\title{
Safety and immunogenicity of a novel multiple antigen pneumococcal vaccine in adults: A Phase 1 randomised clinical trial
}

\section{Claire Entwisle ${ }^{a, *}$}

Claire.Entwisle@Immbio.com

Sue Hill

Yin Pang ${ }^{a}$

Michael Joachim

Ann Mcllgorma

Camilo Colaco ${ }^{\mathrm{a}}$

David Goldblatt ${ }^{b}$

Polly De Gorguette D'Argoeuves

Chris Bailey ${ }^{a}$

almmunoBiology Ltd, Babraham Research Campus, Cambridge, UK

Immunobiology Section, UCL GOS Institute of Child Health, London, UK

${ }^{*}$ Corresponding author.

\section{Abstract}

\section{Background}

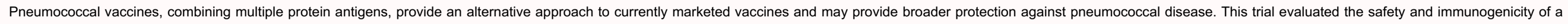
novel vaccine candidate PnuBioVax in healthy young adults.

\section{Methods}

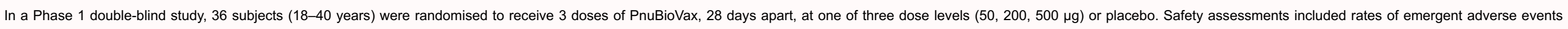
(AEs), injection site and systemic reactions. Immunogenicity endpoints included antibody titre against PnuBioVax and selected pneumococcal antigens. Results

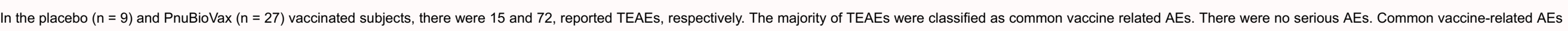

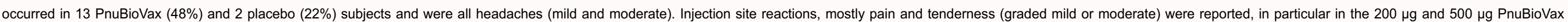

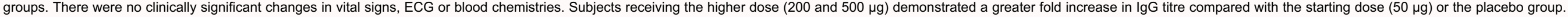

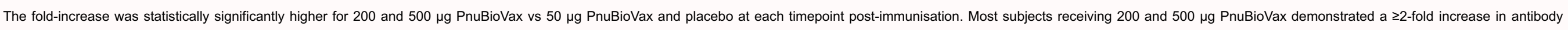
against pneumolysin (Ply), Pneumococcal surface antigen (PsaA), PiaA (Pneumococcal iron acquisition), PspA (Pneumococcal surface protein A) and pilus proteins (RrgB and RrgA).

Conclusions

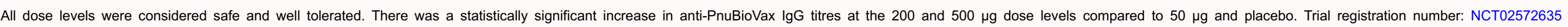
https://www.clinicaltrials.gov. 


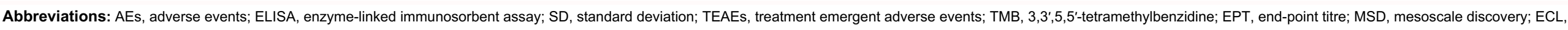

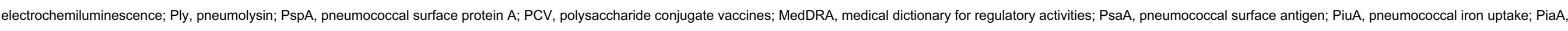
pneumococcalironacquisition; IPD, invasive pneumococcal disease; OM, otitis media; WT, wild type

Keywords: Pneumococcal infection; Phase I; PnuBioVax; Immunogenicity; Safety; Multiple antigen vaccine

\section{Introduction}

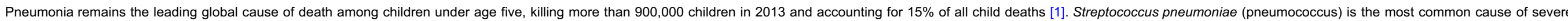

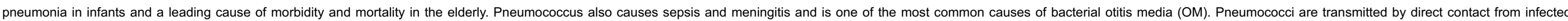

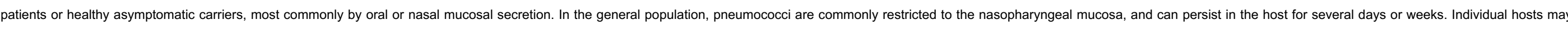

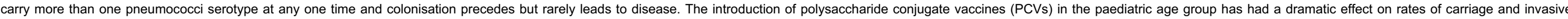

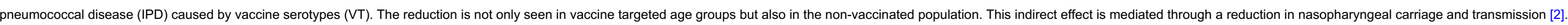

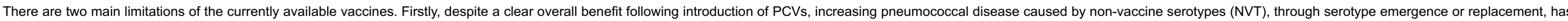

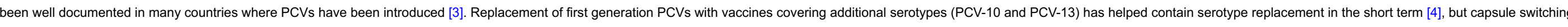

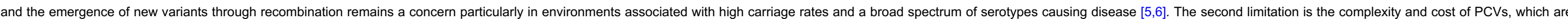
difficult to manufacture and without considerable financial assistance, their affordability and accessibility is restricted for low and middle-income countries [7].

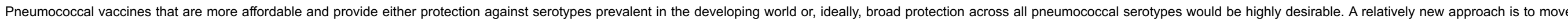

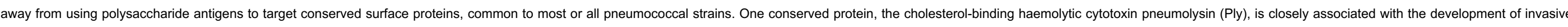

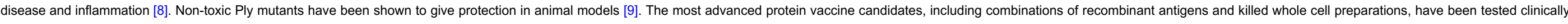

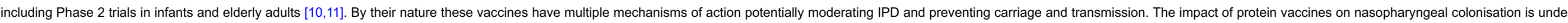
investigation in regions with high carriage rates in Africa [12] and Asia but it remains unclear when these vaccines will progress into larger trials to assess the impact on IPD.

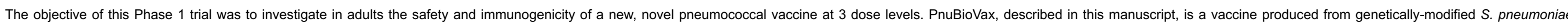

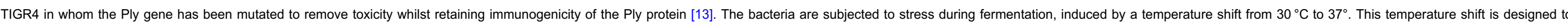

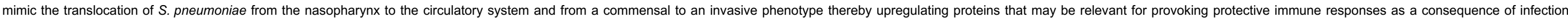

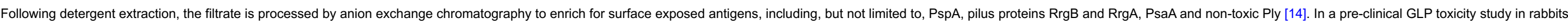

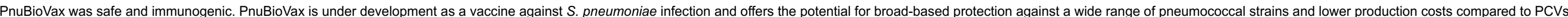

\section{Methodology}

\subsection{Study vaccine}

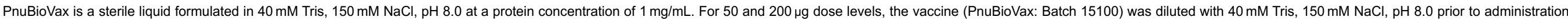

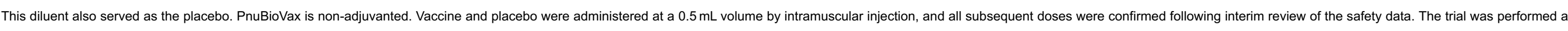
a single site, Simbec Research Limited.

\subsection{Study participants \& study design}

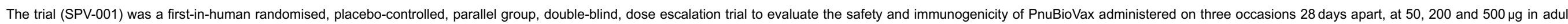

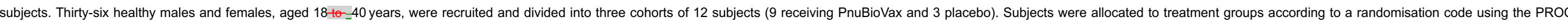
PLAN procedure of SAS® version 9.1.3. 


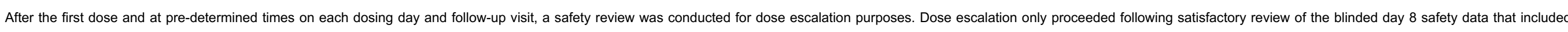
adverse events (AEs), routine laboratory assessments, vital signs, lymph node assessment, injection site reactions and concomitant medication from at least 9 evaluable subjects in the preceding cohort.

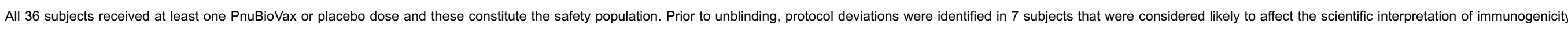
data. Therefore the immunogenicity analysis is based on the per protocol population (29 subjects)

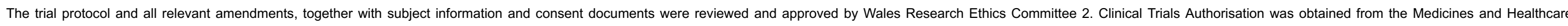
Regulatory Agency (MHRA). The clinical trial (NCT02572635) was performed in accordance with the Declaration of Helsinki (Brazil, 2013) and the principles of Good Clinical Practice

\subsection{Safety and reactogenicity assessment}

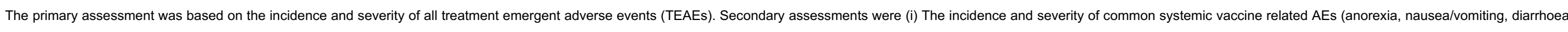

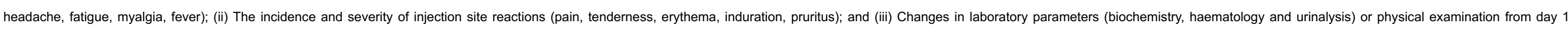
(baseline) to day 64 that were considered to be clinically significant.

\subsection{Immunogenicity assessment}

\subsubsection{Anti-PnuBioVax IgG}

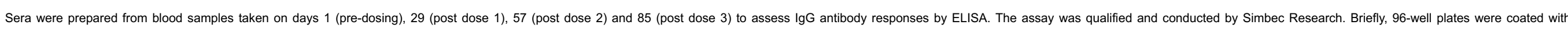

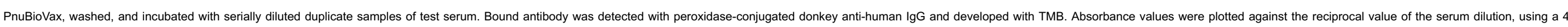
parametric logistic regression with a semi-logarithmic scale to obtain titration curves for each sample. The end-point titre was determined as the titre where the absorbance was equal to a pre-determined cut-point of 0.5 .

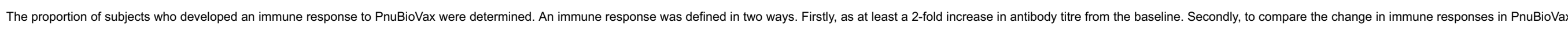
immunised subjects with any change observed in placebo subjects at the same time point. A response was defined as a change in antibody titre that was $\geq 2 \times$ standard deviation (SD) of the placebo change at the same time point.

\subsubsection{Multiplexed electrochemiluminescence (ECL) assay using MSD technology}

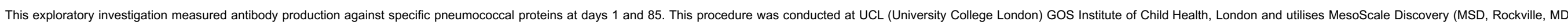

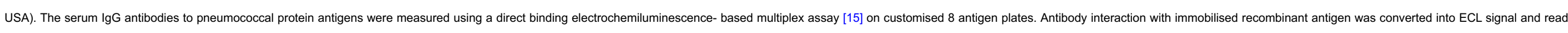
using an ECL detector. ECL signal is relative to antibody concentration.

\subsubsection{Ply neutralisation assay}

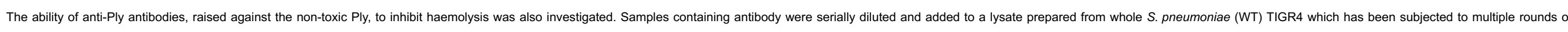

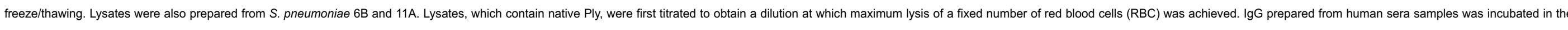

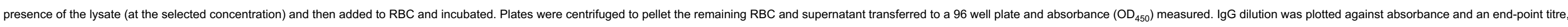
defined as $50 \%$ inhibition of the maximum lysis, obtained.

\subsection{Statistical methods}

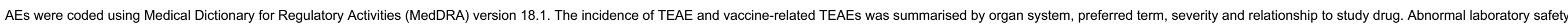

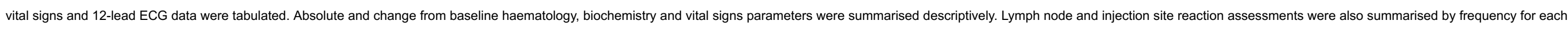
category.

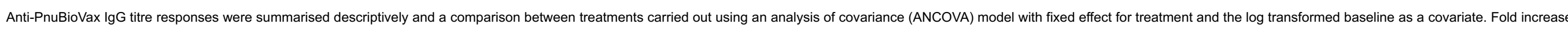

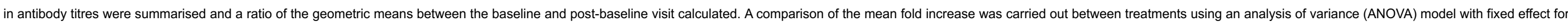


From the fold increase in antibody titres, the proportion of subjects who developed an immune response to PnuBioVax, were summarised by frequency for each response category.

Ply neutralisation titres were compared between treatments using the fold increase in titre between day 85 and baseline samples for each subject and summarised by frequency.

\section{Results}

\subsection{Study participants}

Subject demographics are summarised in Table 1. Of note is the higher percentage of male compared to female volunteers enrolled in the trial.

Table 1 Subject demographics.

\begin{tabular}{|c|c|c|c|c|c|}
\hline Parameter & Statistic & Placebo $(n=9)$ & $50 \mu g$ PnuBioVax $(n=9)$ & $200 \mu \mathrm{g}$ PnuBioVax $(\mathrm{n}=9)$ & $500 \mu g$ PnuBioVax $(n=9)$ \\
\hline \multirow[t]{2}{*}{ Age (yrs) } & Mean & 30.0 & 31.1 & 27.9 & 24.3 \\
\hline & SD & 3.81 & 4.51 & 6.64 & 4.00 \\
\hline \multirow[t]{2}{*}{ Height $(m)$} & Mean & 1.787 & 1.754 & 1.760 & 1.750 \\
\hline & SD & 0.1182 & 0.0914 & 0.0702 & 0.1064 \\
\hline \multirow[t]{2}{*}{ Weight (kg) } & Mean & 85.90 & 77.98 & 73.70 & 76.74 \\
\hline & SD & 16.072 & 13.500 & 9.451 & 14.681 \\
\hline \multirow[t]{2}{*}{$\mathrm{BMI}\left(\mathrm{kg} / \mathrm{m}^{2}\right)$} & Mean & 26.69 & 25.16 & 23.81 & 24.82 \\
\hline & SD & 2.553 & 2.942 & 2.500 & 2.093 \\
\hline Gender: Male & $\mathrm{n}(\%)$ & $7(77.8)$ & $7(77.8)$ & $8(88.9)$ & $6(66.7)$ \\
\hline Female & $\mathrm{n}(\%)$ & $2(22.2)$ & $2(22.2)$ & $1(11.1)$ & $3(33.3)$ \\
\hline Race: Caucasian & $\mathrm{n}(\%)$ & $9(100.0)$ & $9(100.0)$ & $9(100.0)$ & $9(100.0)$ \\
\hline
\end{tabular}

\subsection{Safety and reactogenicity}

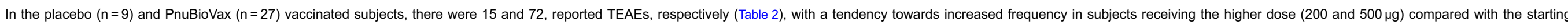
dose $(50 \mu \mathrm{g})$ or the placebo group. The majority of TEAEs were classified as common vaccine related AEs. There were no serious AEs. The common vaccine-related AEs occurred in 13 PnuBioVax (48\%) and 2 placebo $(22 \%)$ subjects.

Table 2 Treatment-emergent adverse events (TEAEs)

\begin{tabular}{|c|c|c|c|c|c|c|}
\hline & Placebo $(n=9)$ & $50 \mu \mathrm{g}$ PnuBioVax $(\mathrm{n}=9)$ & $200 \mu \mathrm{g}$ PnuBioVax $(\mathrm{n}=9)$ & $500 \mu g$ PnuBioVax $(n=9)$ & All PnuBioVax $(n=27)$ & Overall $(n=36)$ \\
\hline Number of TEAEs & 15 & 14 & 24 & 34 & 72 & 87 \\
\hline \multicolumn{7}{|c|}{ Number (\%) of subjects reporting $\geq 1$} \\
\hline TEAE & $6(66.7)$ & $5(55.6)$ & $8(88.9)$ & $8(88.9)$ & $21(77.8)$ & $27(75.0)$ \\
\hline Serious TEAE & $0(0.0)$ & $0(0.0)$ & $0(0.0)$ & $0(0.0)$ & $0(0.0)$ & $0(0.0)$ \\
\hline
\end{tabular}


Number (\%) of subjects with TEAE by severity

\begin{tabular}{|c|c|c|c|c|c|c|}
\hline Mild & $2(22.2)$ & $1(11.1)$ & $4(44.4)$ & $2(22.2)$ & $7(25.9)$ & $9(25.0)$ \\
\hline Moderate & $3(33.3)$ & $3(33.3)$ & $3(33.3)$ & $5(55.6)$ & $11(40.7)$ & $14(38.9)$ \\
\hline Severe & $1(11.1)$ & $1(11.1)$ & 1 (11.1) & $1(11.1)$ & $3(11.1)$ & $4(11.1)$ \\
\hline
\end{tabular}

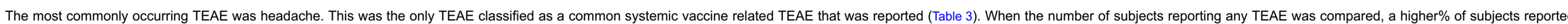

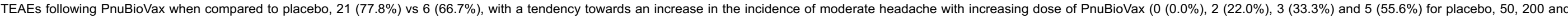
$500 \mu \mathrm{g}$ PnuBioVax, respectively), although the majority were considered unlikely to be or unrelated to the vaccine.Table 4

\section{Table 3 Common vaccine related AEs.}

\begin{tabular}{|c|c|c|c|c|c|c|c|}
\hline \multicolumn{2}{|c|}{ Preferred term } & \multicolumn{6}{|c|}{ Number of subjects (\%) reporting TEAEs } \\
\hline & & Placebo $(n=9)$ & $50 \mu g$ PnuBioVax $(n=9)$ & $200 \mu g$ PnuBioVax $(n=9)$ & $500 \mu g$ PnuBioVax $(n=9)$ & All PnuBioVax $(n=27)$ & Overall $(n=36)$ \\
\hline \multirow[t]{6}{*}{ Headache } & Number of TEAEs & 2 & 3 & 11 & 15 & 29 & 31 \\
\hline & Number (\%) of subjects reporting $\geq 1$ headache & $2(22.2)$ & $2(22.2)$ & $5(55.6)$ & $6(66.7)$ & $13(48.1)$ & $15(41.7)$ \\
\hline & \multicolumn{7}{|l|}{ Number (\%) subjects with headache by severity } \\
\hline & Mild & $2(22.2)$ & $0(0.0)$ & $2(22.2)$ & $1(11.1)$ & $3(11.1)$ & $5(13.9)$ \\
\hline & Moderate & $0(0.0)$ & $2(22.2)$ & $3(33.3)$ & $5(55.6)$ & $10(37.0)$ & $10(27.8)$ \\
\hline & Severe & $0(0.0)$ & $0(0.0$ & $0(0.0$ & $0(0.0)$ & $0(0.0)$ & $0(0.0)$ \\
\hline
\end{tabular}

Table 4 Injection site reactions.

\begin{tabular}{|c|c|c|c|c|c|c|}
\hline \multirow[t]{2}{*}{ Parameter } & \multirow[t]{2}{*}{ Time } & \multicolumn{5}{|c|}{ Number of subjects (\%) reporting any grade of injection site reaction } \\
\hline & & Placebo $(n=9)$ & $50 \mu \mathrm{g}$ PnuBioVax $(\mathrm{n}=9)$ & $200 \mu g$ PnuBioVax $(n=9)$ & $500 \mu \mathrm{g}$ PnuBioVax $(\mathrm{n}=9)$ & All PnuBioVax $(n=27)$ \\
\hline Pain & Days 1-64 & $4(44.4)$ & $7(77.8)$ & $9(100.0)$ & $9(100.0)$ & $25(92.6)$ \\
\hline Tenderness & Days 1-64 & $1(11.1)$ & $4(44.4)$ & $9(100.0)$ & $9(100.0)$ & $22(81.5)$ \\
\hline Erythema & Days 1-64 & $0(0.0)$ & $0(0.0)$ & $0(0.0)$ & $2(22.2)$ & $2(7.4)$ \\
\hline Induration/Swelling & Days $1-64$ & $1(11.1)$ & $0(0.0)$ & $0(0.0)$ & $3(33.3)$ & $3(11.1)$ \\
\hline Pruritus & Days 1-64 & $0(0.0)$ & $0(0.0)$ & $0(0.0)$ & $0(0.0)$ & $0(0.0)$ \\
\hline
\end{tabular}

Injection site reactions (mostly pain and tenderness graded mild or moderate) were commonly reported, in particular in the $200 \mu g$ and $500 \mu g$ PnuBioVax groups. 2 subjects withdrew on the basis of moderate or severe reactions.

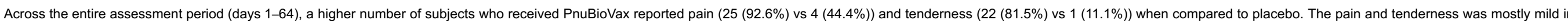
intensity (grade 1) in that it did not interfere with activity and caused mild discomfort to touch.

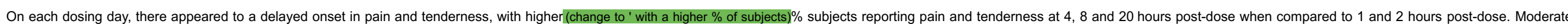

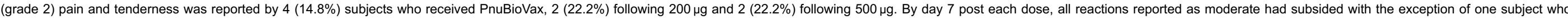
recorded moderate pain and tenderness on day 29 , which progressed to severe on day 31 resulting in them being withdrawn from the trial. 


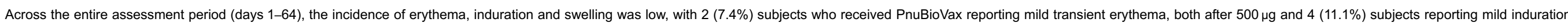

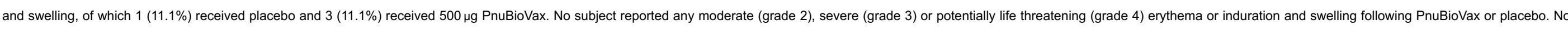
subject reported pruritus at the injection site following PnuBioVax or placebo.

There were no vaccine related clinically significant changes in biochemistry, physical examination, lymph node assessment, vital signs or 12 lead ECG parameters during the trial.

\subsection{Immunogenicity}

\subsubsection{Anti-PnuBioVax IgG titres}

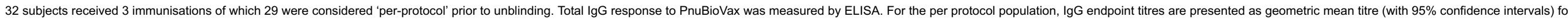

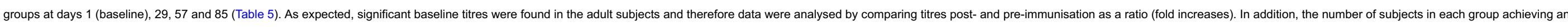
immune response, as defined in Methodology, is reported (Table 5).

Table 5 IgG response to PnuBioVax in per-protocol population.

\begin{tabular}{|c|c|c|c|c|}
\hline & Placebo $(n=9)$ & $50 \mu g$ PnuBioVax $(n=8)$ & $200 \mu g$ PnuBioVax $(n=7)$ & $500 \mu g$ PnuBioVax $(n=5)$ \\
\hline Pre-vac GMT (95\% Cl) Day 1 & $2686.9(1891.2,3817.3)$ & $2343.7(1725.5,3183.2)$ & $2592.4(1597.9,4206.1)$ & $2833.1(1704.8,4707.9)$ \\
\hline Post dose 1 GMT (95\% Cl) Day 29 & $2584.7(1868.8,3575.0)$ & $2524.2(1905.0,3344.7)$ & $3222.2(1956.8,5306.1)$ & $3888.9(2676.2,5651.1)$ \\
\hline GMT ratio & 0.96 & 1.08 & 1.24 & 1.37 \\
\hline Number $(\%)$ subjects $\geq 2$-fold increase & $0(0.0)$ & $0(0.0)$ & $0(0.0)$ & $0(0.0)$ \\
\hline Number $(\%)$ subjects $\geq 2$ SD of placebo fold increase & $0(0.0)$ & $2(25.0)$ & $5(71.4)$ & $4(80.0)$ \\
\hline Post dose 2 GMT (95\% Cl) Day 57 & $2474.5(1778.8,3442.3$ & $2839.2(2135.1,3775.5)$ & $4359.6(2287.1,8310.2)$ & $5448.3(4199.7,7068.3)$ \\
\hline GMC ratio & 0.92 & 1.21 & 1.68 & 1.92 \\
\hline Number $(\%)$ subjects $\geq 2$-fold increase & $0(0.0)$ & $0(0.0)$ & $1(14.3)$ & $2(40.0)$ \\
\hline Number (\%) subjects $\geq 2 \mathrm{SD}$ of placebo fold increase & $0(0.0)$ & $7(87.5)$ & $7(100.0)$ & $5(100.0)$ \\
\hline Post dose 3 GMT (95\%Cl)Day 85 & $2595.4(1889.4,3565.3)$ & $2932.7(2187.7,3931.3)$ & $4742.0(2668.0,8428.4)$ & $6379.9(4886.9,8329.0)$ \\
\hline GMT ratio & 0.97 & 1.25 & 1.83 & 2.25 \\
\hline Number $(\%)$ subjects $\geq 2$-fold increase & $0(0.0)$ & $0(0.0)$ & $4(57.1)$ & $3(60.0)$ \\
\hline Number (\%) subjects $\geq 2 \mathrm{SD}$ of placebo fold increase & $1(11.1)$ & $4(50.0)$ & $6(85.7)$ & $5(100.0)$ \\
\hline
\end{tabular}

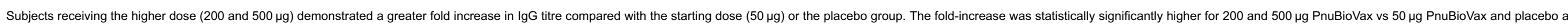

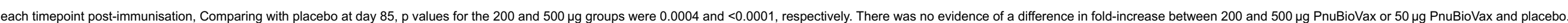

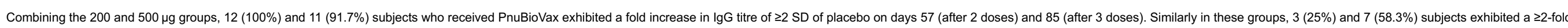
increase in $\lg \mathrm{G}$ titre on days 57 and 85 .

\subsubsection{Antibody responses against pneumococcal proteins}

29 subjects were evaluated in the per protocol population. Antibody responses against pneumococcal antigens PspA, Ply, PsaA, PiaA, PiuA the pilus proteins RrgB and RrgA were measured by MSD assays (Table 6). 
Table 6 Antibody responses against pneumococeal proteins. The number of subjects achieving a $\geq 2$-fold increase from day 1 (baseline) to day 85 in antibody titres against individual pneumococcal proteins.

\begin{tabular}{|c|c|c|c|c|}
\hline \multirow[t]{2}{*}{ Antibody against } & \multicolumn{4}{|c|}{ Number of subjects with $\geq 2$-fold increase $(\%)$} \\
\hline & Placebo $(n=9)$ & $50 \mu \mathrm{g}$ dose $(\mathrm{n}=8)$ & $200 \mu \mathrm{g}$ dose $(n=7)$ & $500 \mu \mathrm{g}$ dose $(\mathrm{n}=5)$ \\
\hline PspA2 & 0 & $1(12.5)$ & $4(57.1)$ & $5(100.0)$ \\
\hline Ply & 0 & $1(12.5)$ & $5(71.4)$ & $3(60.0)$ \\
\hline PsaA & 0 & $0(0.0)$ & $4(57.1)$ & $1(20.0)$ \\
\hline PiaA & $1(11.1)$ & $0(0.0)$ & $5(71.4)$ & $5(100.0)$ \\
\hline PiuA & 0 & $0(0.0)$ & $1(12.5)$ & $0(0.0)$ \\
\hline RrgBT4 & 0 & $8(100.0)$ & $7(100.0)$ & $5(100.0)$ \\
\hline $\operatorname{RrgB6B}$ & 0 & $8(100.0)$ & $7(100.0)$ & $5(100.0)$ \\
\hline RrgAT4 & 0 & $8(100.0)$ & $7(100.0)$ & $5(100.0)$ \\
\hline
\end{tabular}

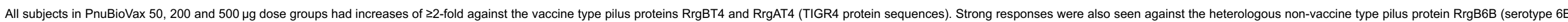

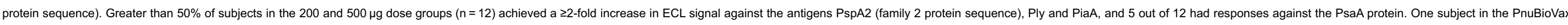
vaccinated groups recorded a $>2$-fold increase in ECL signal against PiuA.

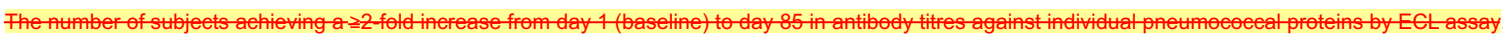

\subsubsection{Ply neutralisation}

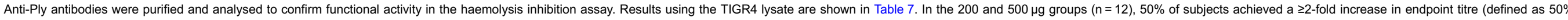

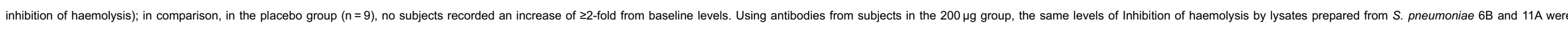
obtained.

Table 7 Number of subjects achieving a $\geq 2$-fold increase from day 1 (baseline) to day 85 in Ply neutralisation titres.

\begin{tabular}{|c|c|c|c|c|}
\hline \multirow[t]{2}{*}{ Anti-Ply response } & \multicolumn{4}{|c|}{ Number of subjects (\%) } \\
\hline & Placebo $(n=9)$ & $50 \mu \mathrm{g}$ dose $(n=8)$ & $200 \mu \mathrm{g}$ dose $(n=7)$ & $500 \mu \mathrm{g}$ dose $(n=5)$ \\
\hline Inhibition of haemolytic activity & $0(0.0)$ & $2(25.0)$ & $4(57.1)$ & $2(40.0)$ \\
\hline
\end{tabular}

\section{Discussion}

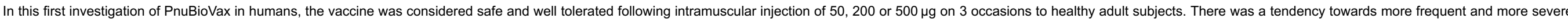

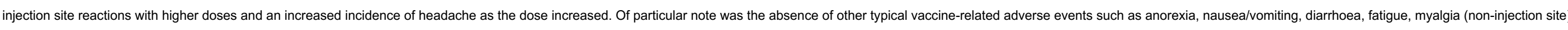
or fever. It can be concluded that vaccine safety would not preclude clinical investigation of PnuBioVax in target populations.

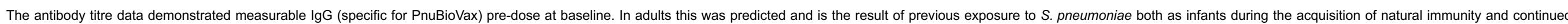

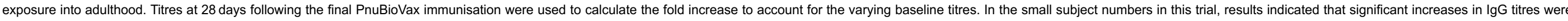
achieved at the 200 and $500 \mathrm{\mu g}$ dose levels. In contrast to polysaccharide-based pneumococcal vaccines, there is no definition of a protective immune response for novel protein-based vaccines, such as PnuBioVax.

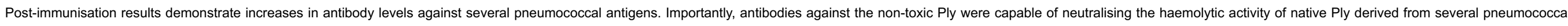
strains. There is evidence that neutralisation of circulating Ply in mice can significantly attenuate cardiac injury during IPD [16]. 


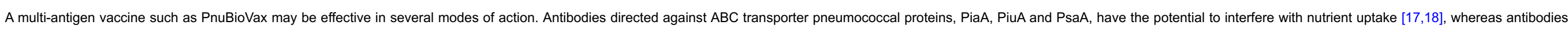

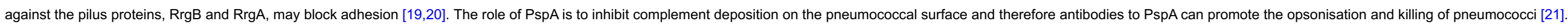

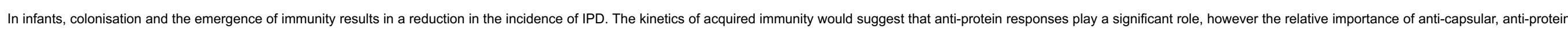

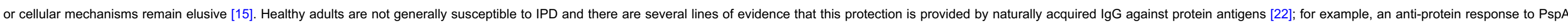
and not a homotypic anti-capsular response was shown to correlate with a reduction in experimental carriage [23].

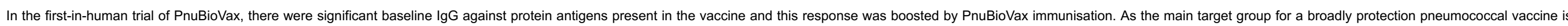
the paediatric population with low natural immunity, the immunogenicity provided by PnuBioVax in this group requires investigation in Phase 2 studies.

\section{Conflict of interest}

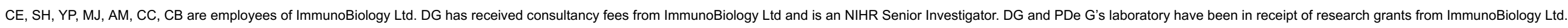

\section{Author's contributions}

$\mathrm{CE}, \mathrm{SH}, \mathrm{MJ}$ and $\mathrm{CB}$ participated in study design, execution of the study, review of data and editing of the manuscript.

\section{Acknowledgements}

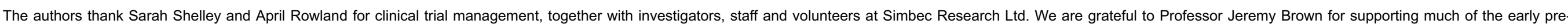
clinical development of PnuBioVax and for advice and encouragement.

\section{References}

[1] K.L. O'Brien, L.J. Wolfson, J.P. Watt, E. Henkle, M. Deloria-Knoll, N. McCall, et al., Burden of disease caused by Streptococcus pneumoniae in children younger than 5 years: global estimates, Lancet 374, 2009 , 893-902.

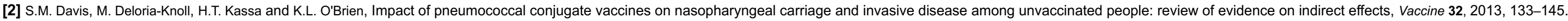

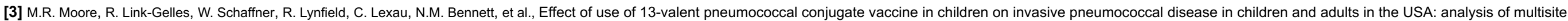
population-based surveillance, Lancet Infect Dis 15, 2015, 301-309.

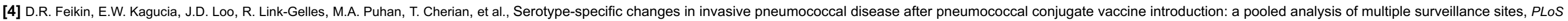
Med 10, 2013, e1001517.

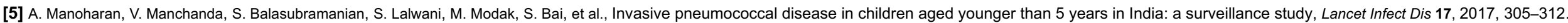

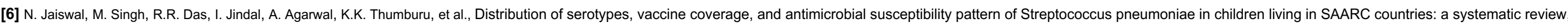
PLoS One 9, 2014, e108617.

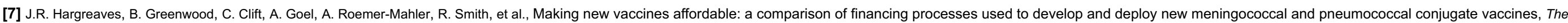
Lancet 378, 2011, 1885-1893.

[8] H.M. Marriott, T.J. Mitchell and D.H. Dockrell, Pneumolysin: a double-edged sword during the host-pathogen interaction, Curr Mol Med 8, 2008, 497-509.

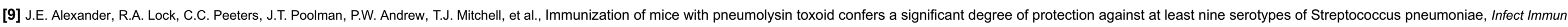
62, 1994, 5683-5688.

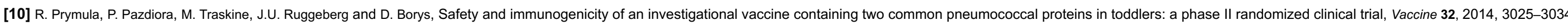

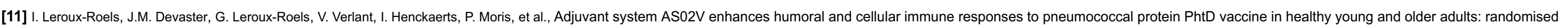




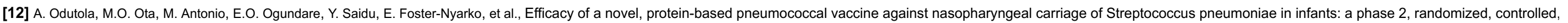
observer-blind study, Vaccine 35, 2017, 2531-2542.

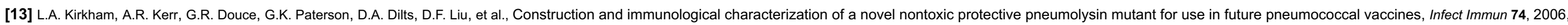
586-593.

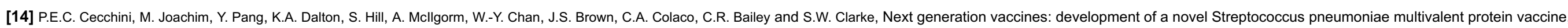
BioProcess J 14, 2015, 18-33.

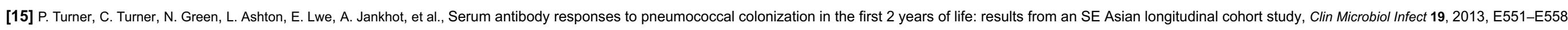

[16] Y. Alhamdi, D.R. Neill, S.T. Abrams, H.A. Malak, R. Yahya, R. Barrett-Jolley, et al., Circulating pneumolysin is a potent inducer of cardiac injury during pneumococcal infection, PLoS Pathog 11, 2015, e1004836.

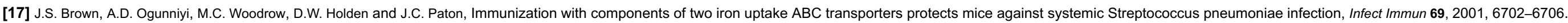

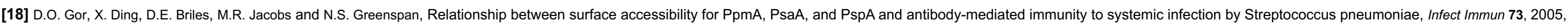
$1304-1312$.

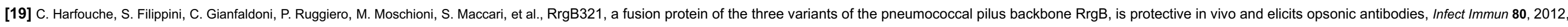
$451-460$.

[20] M. Hilleringmann, F. Giusti, B.C. Baudner, V. Masignani, A. Covacci, R. Rappuoli, et al., Pneumococcal pili are composed of protofilaments exposing adhesive clusters of Rrg A, PLoS Pathog 4, 2008, e1000026.

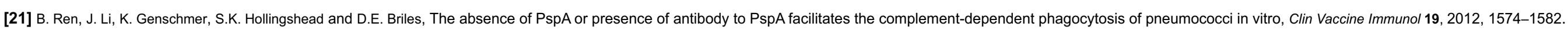

[22] R. Wilson, J.M. Cohen, M. Reglinski, R.J. Jose, W.Y. Chan, H. Marshall, et al., Naturally acquired human immunity to pneumococcus is dependent on antibody to protein antigens, PLoS Pathog 13, 2017 , e1006137.

[23] T.L. McCool, T.R. Cate, G. Moy and J.N. Weiser, The immune response to pneumococcal proteins during experimental human carriage, J Exp Med 195, $2002,359-365$.

\section{Highlights}

- Diseases caused by Streptococcus pneumoniae (pneumococcus) constitute a major global public health problem.

- Pneumococcal vaccines, combining multiple protein antigens, provide an alternative approach to currently marketed vaccines and may provide broader protection against pneumococcal disease.

- In a Phase I clinical trial in healthy adults, PnuBioVax, a novel multiple antigen vaccine was shown to be safe and immunogenic.

\section{Queries and Answers}

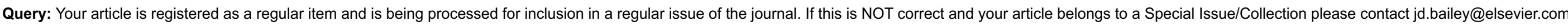
immediately prior to returning your corrections.

Answer:

Query: The author names have been tagged as given names and surnames (surnames are highlighted in teal color). Please confirm if they have been identified correctly.

Answer: 
Query: Highlights should only consist of 85 characters per bullet point, including spaces. The highlights provided are too long; please edit them to meet the requirement.

Answer:

Query: Please check the hierarchy of the section headings.

Answer: 